\title{
Experiências sobre Imunização e o Papel da Atenção Primária à Saúde
}

\author{
Priscilla Azevedo Souza, Beatriz Gandra, Ana Cláudia Cardozo Chaves
}

\section{RESUMO}

Este artigo apresenta uma análise das experiências de imunização submetidas ao Prêmio APS Forte apontando seus desafios e praticas na condução de uma das mais relevantes e consolidadas intervenções em saúde pública no Brasil.

Palavras-chave: Atenção Primária à Saúde; Acesso; Prêmio APS.

\section{ABSTRACT}

This article presents an analysis of the immunization experiences submitted to the APS Forte Award, pointing out its challenges and practices in conducting one of the most relevant and consolidated public health interventions in Brazil.

Keywords: Prmary Health Care; Access; PHC Prize.
Revista da Rede APS 2020

Publicada em: 04/09/2020

DOI:10.14295/aps.v2i3.57

Priscilla Azevedo Souza

(Ministério da Saúde, Brasil)

Beatriz Gandra

(Ministério da Saúde, Brasil)

Ana Cláudia Cardozo Chaves (Ministério da Saúde, Brasil)

Correspondência para:

Priscilla Azevedo Souza priscilla.souza@saude.gov.br 


\section{INTRODUÇÃO}

A vacinação é considerada uma das mais relevantes e consolidadas intervenções em saúde pública no Brasil. Representa a principal estratégia de promoção e proteção da saúde, prevenção das doenças imunopreveníveis, e o procedimento de menor custo e maior efetividade.

O Ministério da Saúde, por meio do Programa Nacional de Imunizações (PNI) oferece, no Calendário Nacional de Vacinação, todas as vacinas recomendadas pela Organização Mundial de Saúde, além de imunobiológicos especiais de alto custo financeiro, para grupos em situação de maior risco, com um rígido controle de qualidade que segue os padrões dos mais eficientes e confiáveis programas de vacinação do mundo.

A Atenção Primária à Saúde (APS) é o âmbito da atenção mais estratégico para a prevenção de doenças e agravos, sendo um dos seus atributos essenciais o acesso de primeiro contato para os usuários do Sistema Único de Saúde (SUS). Assim, na perspectiva do controle, erradicação e eliminação de doenças imunopreveníveis, o que inclui as ações de imunização, é fundamental a participação ativa dos profissionais de saúde que atuam na APS, bem como de gestores municipais e estaduais de saúde.

Nas Unidades Básicas de Saúde ou Unidades de Saúde da Família, a vacinação de rotina deve ser realizada em conformidade com as normas do PNI, segundo o calendário de vacinação estabelecido pelo Ministério da Saúde. É importante que profissionais da saúde, gestores municipais e estaduais, das áreas da Atenção Primária e da Vigilância em Saúde, estejam integrados e desenvolvam estratégias de acordo com as necessidades de seu território para o alcance da metas das coberturas vacinais.

Por ser uma das principais ações estratégicas do Governo Federal, a vacinação consta na lista da Carteira de Serviços da APS (CaSAPS, 2019), que visa nortear a prática clínica nas unidades de saúde, além de ser um dos indicadores de pagamento por desempenho do financiamento da APS. As três esferas de governo devem estar alinhadas para o desenvolvimento de estratégias mais eficazes voltadas para a imunização, para que seja possível erradicar as doenças imunopreveníveis do nosso país.

\section{ANÁLISE DAS EXPERIÊNCIAS}

Obteve-se um total de 51 experiências de todas as regiões do Brasil sobre a temática de imunização, sendo 19 do Sudeste, 14 do Sul, 12 do nordeste, 2 do Norte e 4 do Centro-Oeste. Foram 23 escritas por equipes de Saúde da Família, 10 de Secretarias Municipais de Saúde, 2 de Secretarias Estaduais de Saúde, 6 de Coordenações ou Gerências de APS e 10 de outros locais, como Coordenações de Imunização e Vigilância epidemiológica. A maioria das experiências teve início de 2017 a 2019.

Um grande desafio enfrentado pela saúde em todo o território nacional é a baixa cobertura vacinal. Com a imprescindibilidade de minimizar esse problema, profissionais da saúde e gestores municipais e estaduais reorganizaram seus processos de trabalho, desenvolveram estratégias inovadoras e reforçaram ações já conhecidas, e assim obtiveram resultados positivos na ampliação da cobertura de imunização.

Os atributos do acesso/primeiro contato e da orientação comunitária foram identificados com maior frequência nas experiências exitosas para Imunização. Alguns municípios utilizaram como estratégia a ampliação do horário de funcionamento da sala de vacina para que a população dispusesse de mais opções de horários para atualizar a situação vacinal, em especial a população que trabalha em horário comercial e não tem possibilidade de visitar as unidades de saúde durante a jornada de trabalho.

Também foram identificados os atributos Integralidade e Longitudinalidade durante a análise das experiências, onde foram realizadas estratégias como a vacinação de rotina, com um olhar holístico para o cidadão durante a 
consulta, verificando o seu cartão vacinal independe do motivo que o levou a unidade de saúde. Os profissionais dispuseram de outras oportunidades para verificar a situação vacinal, como grupos terapêuticos, atividades do Programa Saúde na Escola, entre outros.

A maioria das experiências apresentaram somente uma estratégia para o aumento da cobertura vacinal, como: verificações frequentes nos cartões de vacina de um públicoalvo, verificações frequentes na ficha espelho, visitas nos domicílios no território, vacinação extramuros em escolas e estações de metrô, variados meios de comunicação para sensibilizar a população sobre a importância da imunização, entre outras. Outras experiências referiram realizar parceria com a Vigilância Epidemiológica, Vigilância Sanitária e Secretaria Municipal de Educação para traçar ações estratégicas de acordo com as necessidades do território.

A educação em saúde foi uma estratégia muito utilizada nessas experiências, por ser considerada uma ação fundamental na APS, em especial quando trata-se do tema imunização. Alguns relatos referiram realizar ações de educação em saúde sobre a vacinação, enfatizando sua importância em todas as fases da vida e as consequências acarretadas pela não imunização. Essa estratégia ampliou a cobertura vacinal, além de sensibilizar e estimular o autocuidado da população.

A partir da análise das experiências na APS, destaca-se como inovação a criação de instrumentos para monitorar a cobertura vacinal da população em âmbito municipal, como é o caso do Vacinômetro. O Vacinômetro foi criado a partir de uma planilha alimentada diariamente com todas as vacinas do PNI e com informações de toda a população do município de forma estratificada, assim os profissionais saberão qual população deve ser alvo de intensificação da vacinação, além da geração de indicadores para traçar planos de enfrentamento dos agravos imunopreveníveis.

Outras ações relatadas em relação à busca ativa de crianças com esquema vacinal incompleto e à ampliação do horário de funcionamento das salas de vacina nas unidades, não são exatamente inovadoras, mas se destacam como formas de facilitação do acesso às ações de imunização disponíveis nas unidades de APS que demandam muito pouco em relação à infraestrutura e insumos, sugerindo que o compromisso e a disponibilidade das equipes de saúde para adaptações relativamente simples no processo de trabalho podem promover mudanças com efeito positivo sobre a proteção imunológica da população.

As experiências municipais que demonstraram maior efeito na ampliação da cobertura vacinal foram as que utilizaram estratégias combinadas para o aumento da cobertura. Essa tendência confirma o que referem evidências internacionais quanto à eficácia de intervenções sobre imunização que envolvem múltiplos componentes, especialmente aquelas que envolvem a comunidade (CROCKER-BUQUE et al, 2017).

Nesse sentido, um pouco mais de $20 \%$ das experiências analisadas utilizaram a combinação de ações como: monitoramento dos cartões de vacina, busca ativa de faltosos, bloqueio vacinal, educação em saúde, estratégias de comunicação para sensibilização da população, vacinação extramuros, ampliação do horário de funcionamento, capacitação dos profissionais de saúde, entre outros.

Foi apontada como uma das estratégias mais problemáticas dentre as experiências avaliadas a imposição de barreiras de acesso a algum tipo de serviço em função da atualização do esquema vacinal. São exemplos disso, condicionar a frequência na escola/creche mediante cartão de vacina atualizado ou vincular a frequência em um grupo de educação em saúde para pais/responsáveis mediante cartão de vacinação completo de crianças. Os que estivessem sem cartão ou com o cartão desatualizado, não poderiam participar das atividades.

Considerando as experiências relatadas como exitosas, elas estão relacionadas a: Impacto das ações de monitoramento na cobertura vacinal; Ações de educação em saúde para a população para ampliação da cobertura vacinal; 
Organização do funcionamento por bloco de hora como estratégia para ampliar acesso; Vacinação de rotina- inserção da vacina no processo de trabalho; Busca ativa utilizando variados meios de comunicação; Agendamento online para realização da vacina; Estratégias em conjunto com PSE; Vacinação extramuros; Bloqueio vacinal, varredura e busca ativa; Educação em saúde realizada por professores com apoio dos profissionais de saúde; Variados meios de comunicação para campanha de vacinação e sensibilização da população; Implantação da rede de imunização do município; Ações de educação em saúde e meio lúdico para sala de espera; Monitoramento da cobertura vacinal por meio das ficha espelho e consulta aos cartões durante a rotina; Parceria da SMS com SVS para ampliar cobertura; Parceria SMS com SME; Muitos utilizaram variadas estratégias para buscar melhor resultado nas ações e ampliar cobertura; Criação de um instrumento para monitorar cobertura vacinal (Vacinômetro); Intensificação de vacinação em público alvo.

\section{DISCUSSÃO}

A adesão a essa ação preventiva é fundamental, especialmente no primeiro ano de vida, pois contribui para o decréscimo da morbidade e da mortalidade causadas pelas doenças infecciosas evitáveis no público infantil. Muitas crianças deixam de ser vacinadas pelos mais diferentes fatores, que abrangem nível cultural e econômico, crenças, superstições, mitos e credos religiosos.

As coberturas vacinais vêm caindo no país e doenças até então eliminadas estão reincidindo. Diante desta situação, faz-se necessária a implementação de estratégias para aumentar a cobertura vacinal, principalmente em regiões isoladas, áreas mais vulneráveis e locais que apresentam barreiras ao acesso para aqueles que querem ser vacinados, ou vacinar seus filhos. Nesse sentido, este instrumento pretende contribuir para o fortalecimento das ações desenvolvidas pelas equipes de APS para o alcance das metas de coberturas vacinais.
A organização do processo de trabalho da APS integrada aos demais pontos da rede de atenção constitui forte aliada no enfrentamento dos principais problemas de saúde e eficiência, efetividade e qualidade das ações propostas.

As experiências dialogam com os dez passos divulgados pelo Ministério da Saúde para os trabalhadores da APS garantir a ampliação das coberturas vacinais nas unidades: 1 - manter a sala de vacina aberta todo o horário de funcionamento da unidade; 2 - evitar barreiras de acesso; 3 - aproveitar as oportunidades de vacinação em consultas ou outros procedimentos na unidade; 4 - monitorar a cobertura vacinal e realizar busca ativa de usuários faltosos; 5 - garantir o registro adequado da vacinação no cartão de vacinação do usuário e nos sistemas de informação (e-SUS AB); 6 - orientar a população sobre atualização do calendário vacinal; 7 - combater qualquer informação falsa sobre vacinação; 8 - intensificar as ações de vacinação em situações de surto; 9 promover a disponibilidade e a qualidade das vacinas ofertadas à população; 10 - garantir pessoal treinado e habilitado para vacinar durante todo o tempo de funcionamento da unidade.

As experiências que obtiveram maior impacto na ampliação da cobertura vacinal foram as que utilizaram estratégias combinadas. Um estudo realizado no Ceará, após uma das maiores epidemias de sarampo do estado entre 2013 e 2015, reforça a utilização de estratégias combinadas para prevenir a população de dezenas de doenças por meio da imunização. Após a realização de pelo menos 4 estratégias concomitantemente, foi alcançado $95 \%$ de cobertura vacinal no estado do Ceará (MOURA et al, 2018).

Importante destacar o aspecto multiplicador de algumas experiências, que podem ser replicadas por outros profissionais ou gestores em outras localidades. Assim como é fundamental para sustentabilidade das experiências ou que os profissionais já envolvidos nas experiências possam dar continuidade e ter o apoio da gestão, garantindo assim a longitudinalidade da atenção. 
A integração entre as bases de dados do Sistemas de Informação em Saúde para a Atenção Básica (SISAB) da estratégia e-SUS Atenção Básica (e-SUS $A B$ ) e do Sistema de Informação do Programa Nacional de Imunizações (SIPNI) qualifica o processo de gestão da informação e fomenta melhoria nas ações de monitoramento, avaliação e tomada de decisão para o alcance de bons resultados em relação à imunização.

\section{CONSIDERAÇÕES FINAIS}

Reconhecendo a importância da imunização como forte instrumento de proteção à saúde, torna-se fundamental o desenvolvimento de ações que ampliem e promovam a vacinação. $A$ necessidade da ampliação da cobertura intensificou a vacinação em todo o Brasil, o que permitiu a aplicação de práticas já conhecidas, como a vacinação de rotina e estratégias de educação em saúde, mas também possibilitou o desenvolvimento de ações inovadoras, como instrumentos de monitoramento da cobertura e a abertura da sala de vacina em horários alternativos aos tradicionais. como as relatadas....

Os profissionais e gestores municipais e estaduais demonstram empenhar esforços para atingir as metas de imunização com qualidade e segurança. $O$ enfrentamento de grandes epidemias está alinhado ao sucesso das campanhas de multivacinação, integrada na rotina dos serviços da APS, bem como aperfeiçoamento dos processos de imunização e contexto local.

Considerando a relevância dessa temática no âmbito da saúde pública, destaca-se a necessidade de intensificação de novas estratégias, bem como o registro e publicação de experiências bem sucedidas já realizadas, para que seja possível a replicabilidade em outros locais e assim, progressivamente, a ampliação da imunização na população brasileira. 


\section{NOTAS E REFERÊNCIAS}

Crocker-Buque, T., Mindra, G., Duncan, R. and Mounier-Jack, S., 2017. Immunization, urbanization and slums-a systematic review of factors and interventions. BMC Public Health, 17(1), p.556.

Moura, A.D.A., Carneiro, A.K.B., Braga, A.V.L., Bastos, E.C.D.S.A., Canto, S.V.E., Figueiredo, T.W.S., Garcia, M.H.D.O., Lemos, D.R.Q. and Andino, R.D., 2018. Estratégias e resultados da vacinação no enfrentamento da epidemia de sarampo no estado do Ceará, 2013-2015. Epidemiologia e Serviços de Saúde, 27.

Pires-Oliveira, T. and Souza-Machado-Oliveira, L., 2020. La inmunización de niños en Brasil: panorama jurídico y reflexión bioética. Revista de Bioética y Derecho, (48), pp.227-243. 